\title{
A Plastic, Dynamic and Reducible 3D Geometric Model for Simulating Gramineous Leaves
}

\author{
Christian Fournier \\ INRA \\ UMR 759 LEPSE \\ F-34090 Montpellier, France \\ christian.fournier@supagro.inra.fr
}

\author{
Christophe Pradal \\ CIRAD \\ CIRAD/INRIA/INRA EPI VirtualPlants, UMR AGAP \\ 34398 MONTPELLIER CEDEX 5, France \\ christophe.pradal@inria.fr
}

\begin{abstract}
Unlike trees, the 3D architecture of gramineous plants is much more related to the shapes of its leaves than the arrangement of its branches. Many modelling efforts have thus concentrated on correctly capturing its complex shape at different stages and use them as scalable geometric primitives. Still, additional control of such objects is needed in the context of Functional Structural Modelling. The objective of this work is to propose a plastic and dynamic 3D leaf model that is well suited for such uses, still able to capture a variety of observed static shapes. Leaf shape is modeled by a parametric surface describing leaf midrib curvature, leaf width variation, undulation of leaf margins and twist along the midrib. Meshes can be generated from these surfaces, and reduced using a decimation algorithm. The model can be fitted with data or with curves drawn by user interaction. Morphological operators are defined and allows for plastic deformation of the control curves. The dynamics of shape acquisition can also be specified, and combined with morphological operators to simulate various scenarios of evolution and responses to stresses. The capabilities of the model are demonstrated through several cases of use. Future directions of research are thought to be a better integration of mechanical or physiological constraints that would reduce the model plasticity but avoid user-induced unrealistic simulation.
\end{abstract}

Keywords-geometry; leaf; graminae; dynamic; plasticity; decimation; FSPM

\section{INTRODUCTION}

3D geometry defines the interface of plants with the environment and is responsible for a great part of the visual realism of 3D reconstruction. For some plants, like trees, geometric models can concentrates on arrangement of branches and allometries between them without paying too much attention to the leaves, and obtain very realistic looking trees [1], [2]. Such an approach would on the contrary yield bad results on plants such as graminae where 3D architecture relies less on the arrangement of axes than on the individual shapes of leaves. This stands for representing plant shape at a given moment in time, but also for capturing its dynamic evolution during ontogeny or its changes in response to environmental stresses.

The geometry of the gramineous leaf and its dynamics can be decomposed in several parts that are determined by

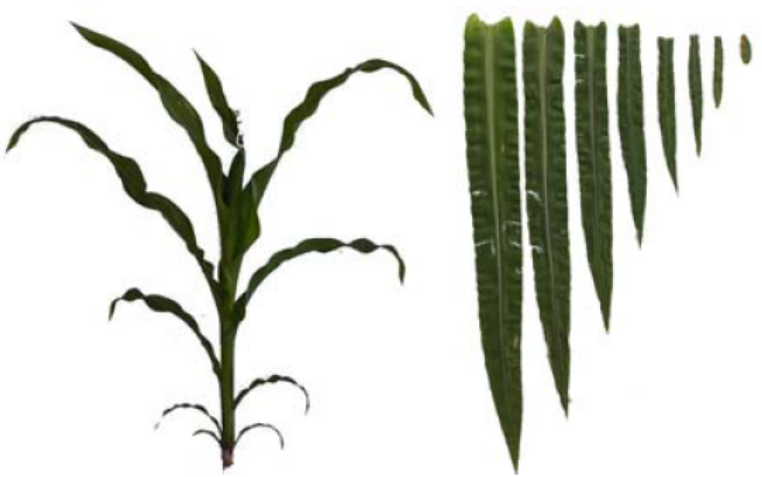

Figure 1. Morphology of gramineous leaves exemplified by a growing maize plant (left) and top view of detached planified leaves (right). Leaf geometry can be decomposed in leaf width variation along the blade (right), leaf curvature at the midrib, and the undulation of margins. The top of the plant is composed by a whorl of rolled leaves, that modify their curvature.

different processes (Figure 1).

First, meristematic activity at the base of the growing leaf results in leaf enlargement and extension, the balance between the two determining the variation of leaf width as a function of distance to leaf base [3]. This balance evolves during ontogeny and can be altered by the environmental conditions, especially light availability and $\mathrm{N}$ contents [4], [5]. On some species, like maize, meristematic activity in not homogeneous in the entire leaf section, and produces margins that have not the same length as the midrib region. This results in undulations at the leaf margins, or twisting of the principal plane of the leaf [6]. Second, the complex set of mechanical constraints that are exerted on leaf tissues during their maturation, together with the rate of tissue rigidification, will determined the local curvature of the leaf. This local component has been found to be the principal factor on maize leaves, the gravity only deforming to a small amount this rigid structure [7]. The dynamic of leaf curving during growth is additionally determined by the dynamics of leaf unrolling, which in turns depends of the unrolling rate of surrounding leaves in the whorl. Occasionally and for 
short period, the leaf curvature can be strongly altered by stresses that induce rolling and straightening of leaves [8], [9]. Finally, gramineous leaves have at their base a tissue, the auricle, which can change its shape during ontogeny or in response to external conditions and actively modify leaf angle.

Embracing in a mechanistic model all this complexity is currently out of reach, and most of leaf geometric models are empirical and static. Still, some of the dynamic processes determining leaf shape, like leaf extension rate or the intensity of stresses, can be quantitatively predicted by functional models. Linking empirical static geometric models to dynamical variables in a realistic way may thus be challenging. In a 3D simulation, the level of geometrical details may also depend on the finality of the application. A detailed leaf (with undulation or twist) is needed for rendering realistic scenes, while simplified, still accurate, representation can help saving substantial computation time for e.g. light computation. A geometric model has thus to be modular enough to represent all the type of geometrical shapes, and perform efficient simplifications. The objective of this work is to propose an integrated solution for geometric modeling of gramineous leaves. We aim at building a plastic and dynamic 3D leaf model, capable of mimicking leaf dynamics but also able to simulate the elastic or nonelastic responses to stresses. We also conceived the model to capture a variety of shapes with different level of details and with capabilities of simplification in simulation. Finally, the model comes with methods for assimilation of usual static experimental data and derivation of dynamic parameters. To ease the diffusion of this model and allow its integration in several virtual gramineous plants, we design it as a software component available on the OpenAlea plateform [10].

\section{RELATED WORK}

The gramineous leaf geometry has been mathematically described for several aspects. The leaf width variation has been modeled, as a function of the distance to leaf base, by a polynomial [11], [12], [13], by modified polynomial function [14], or by a composition of functions reflecting leaf growth stages [15]. Leaf midribs have been described as arc of circle [16], arc of conics [13] or quadratics [17]. Leaf margin undulations have been proposed to be cycloid [18], [19], [20], [21]. These models are very compact and parsimonious, and give the user access to standard mathematical tools for modeling leaf shape transforms or analyzing derivatives. They have been used to reconstruct 3D plants from digitised partial data [22], [23], [20] or in models that concentrate on leaf extension [24], [25], [26], [27], [28]. In such cases, unknown parameters are considered as random variables that follow pre-defined or measured statistical laws [13], [20], [17]. Parameters could also be used for quantifying the genetic variability of leaf shapes [29]. This approach may however induced some inconsistency in the shapes produced, because they do not take into account the co-variation of parameters, and have a strong intrinsic constraint in the type of curve they can fit. For instance, the mathematical representation of the midrib can not represent a leaf midrib with inflection points.

A more flexible approach has been used to get 3D reconstructions of any type of leaves. The idea is to use smoothing function to build in a first step atlases of 3D shapes and use them, after appropriate scaling, to construct the plant.

This includes the use of smoothing spline [30], [23], [27] or hermit curves [31] that can be used to control advance graphics primitives like generalised cylinders [32]. In some cases, digitised mesh are directly used as scalable geometric objects [33], [34]. The drawback of the later method is that the modeler looses part of the control facilities offered by mathematical or control functions for operating on leaf shapes.

The methods described above stands for reconstructing plants at a given point in time (snapshots). The problematic is a bit different for dynamic simulations. First, dynamic simulation can be obtained by producing every time step a static reconstruction, possibly with evolving pre-defined shapes [16], [33], [35]. This approach has the advantage of simplicity, and gives satisfactory results at the canopy scale. Still, it does not allow predicting realistic patterns of shape evolution at the individual leaf or plant scale, as the system has no memory of the previous state. For example, re-scaling a pre-defined leaf object to account for a stress will effectively produce a smaller leaf, but with a completely new shape locally. Such metamorphoses during leaf ontogeny are embarrassing for the production of realistic animation of plant development. Also, in cases where leaf shape modification has an effect on plant growth (e.g. via reduced interception of light), the model will not correctly simulate the dynamics of differentiation of plants within the canopy. Finally, by definition, pre-defined leaf shapes are not predictive, and thus could not be used for its validation.

To solve these issues, more realistic, locally controlled, scenarios of individual leaf shape acquisition have been proposed. [24] and [36] used pre-defined mature shapes to build a dynamical scenario of evolution that truncate leaf width profile and use the mature leaf curvature as a trajectory for leaf midrib during extension. [37] proposed a set of rules for building growing leaves in the whorl as intermediate shapes between straight vertical ones and the shape of the first mature leaf. [38] defined a set of morphological operators that evolves with time and allow smooth simulation of individual leaf growth.

Whilst being compatible the every time step reconstruction strategy, our model clearly ambition to improve and ease the realistic modeling of individual leaf shape dynamic using time dependant operators. We also keep the idea of controlled curves, a method that both allow to fit to a large 
range of data and keep mathematical control of the shape during growth.

\section{MODEL DESCRIPTION}

\section{A. Equation of the surface}

Let $\mathcal{N}$ be a $2 \mathrm{D}$ curve representing the planar leaf midrib, and $r$ be a function from $[0,1]$ representing the leaf width variation. The leaf shape is represented as a parametric surface constructed by sweeping a segment of length $r(s)$ along the midrib curve $\mathcal{N}$. The segment is orthogonal to the plane of the midrib curve.

Let $\mathcal{N}: s \mapsto(x(s), 0, y(s))$ and $r: s \mapsto r(s)$ be two functions from $[0,1]$ to $\mathbb{R}^{2}$ and $\mathbb{R}$ respectively. The absolut frame is defined as $(O, \vec{i}, \vec{j}, \vec{k})$. Let $\mathcal{R}$ be a rotation frame, also called moving frame [39], along a curve. The parametric surface obtained by sweeping a centered segment of length $r(s)$ along the midrib can be written as:

$$
S(s, u)=\mathcal{N}(s)+\mathcal{R}(s) P(s, u)
$$

If we consider $\mathcal{R}(s)$ the identity matrix and $\mathcal{P}: s, u \mapsto$ $r(s) u \vec{j}$ a segment of length $r(s)$, then the equation is equivalent to :

$$
\begin{aligned}
& \forall s \in[0,1], \forall u \in[-0.5,0.5] \\
& S(s, u)=(x(s), r(s) u, y(s))
\end{aligned}
$$

To represent the twist of the leaf, the segment can be rotated along the tangent of the curve. The analytical form becomes more complex but quite similar to the previous one. In the same way, marginal undulations can be modelled by a different cycloid for each side of the leaf.

In the following equation, the segment is rotated around the $y$ axis $(\vec{j})$ of an angle $\theta(s)$ :

$$
\mathcal{R}: s \mapsto(\cos (\theta(s)), 0, \sin (\theta(s)))
$$

The equation becomes:

$$
\begin{aligned}
S(s, u)= & (x(s)+r(s) \cos (\theta(s)) u, \\
& r(s) u, \\
& y(s)+r(s) \sin (\theta(s)) u)
\end{aligned}
$$

Finally, the general form of the equation is:

$$
S(s, u)=(x(s)+f(s, u), h(s, u), y(s)+g(s, u))
$$

with $f, g$, and $h$ three functions that allow to represent several effects such as twist and undulation.

\section{B. Fiting the model with data}

Irrespective of the targeted application (static reconstruction or dynamical simulation) the model has to be fitted with data describing one or several steps of leaf midrib curvature evolution and profile of leaf width variation. In both cases, these data are normalised, in order to ease subsequent re-scaling for representing different leaves or link to physiological age.

The data can be obtained directly from leaf scans and digitization [40], [41], or from already modeled shapes, by sampling modeled curves at small intervals. Data are stored as collections in an indexed atlas, to allow for organizing variation with leaf rank and/or leaf ontogeny.

Building the parametric surface requires to be able to interpolate $x, y$ and $r$ along the midrib at any distance from leaf base. To do so, we first unify the parametrisation of the three variables as a function of $s$, and fit a smoothing spline for each. Starting from raw $\left(x_{i}, y_{i}\right)$ data points, corresponding curvilinear abscissa $s_{i}$ comes with :

$$
s_{i}=\sum_{j=1}^{i} d s_{i}=\sum_{j=1}^{i} \sqrt{\left(x_{i}-x_{i-1}\right)^{2}+\left(y_{i}-y_{i-1}\right)^{2}}
$$

As $r$ is naturally expressed as a function of $s$, it is easy to get all three function fully parametrised at all $s_{i}$. The normalisation of the curve is done both for length and leaf surface. Normalising midrib $\mathcal{N}$ by leaf length $L$ gives:

$$
\forall s \in[0, L], \mathcal{N}^{\prime}(s)=\left(\frac{x\left(\frac{s}{L}\right)}{L}, \frac{y\left(\frac{s}{L}\right)}{L}\right)
$$

That is after operating a simple variable change, we obtained:

$$
\forall s^{\prime} \in[0,1], \mathcal{N}^{\prime}\left(s^{\prime}\right)=\left(\frac{x\left(s^{\prime}\right)}{L}, \frac{y\left(s^{\prime}\right)}{L}\right)
$$

Moreover, on normalised leaves, the leaf surface is given by:

$$
\text { surface }_{\text {leaf }}=\int_{0}^{1} r(t) d t
$$

To get this surface equal to unit, $r(s)$ is thus normalised to:

$$
\forall s \in[0,1], r^{\prime}(s)=\frac{r(s)}{\int_{0}^{1} r(t) d t}
$$

\section{Dynamic equation of the surface}

A dynamic equation of the leaf surface can be obtained from one $r(s)$ function combined with several midrib curves $\mathcal{N}^{i}(s)$ describing the temporal evolution of the curvature. To do so, data are normalised and leaves reconstructed using physiological age ([42]). Physiological age represents the progress of leaf in its development. For simulation of plants where mature length is known, this age can be approximated by $\frac{l}{L}, l$ being the current leaf length and $L$ the mature leaf length. Normalised $r(s)$ gives $r(a g e)$. For leaf curvature, we considered a collection of normalised shapes $\mathcal{N}($ age, $s)$. 
For reconstruction, $\mathcal{N}(s)$ at a given age is obtained by interpolating the different curves. The surface at $t$ is given by progressing from the top of the leaf to the base.

Let $L$ be the expected mature length, $R$ be the expected maximal width, $r_{\max }$ the maximum of $r(s)$ and $l$ the current leaf length. The surface is dependent of an unknown $h$ :

$$
\begin{aligned}
& \forall s \in[0, h], \forall u \in[-0.5,0.5], \\
& S^{\prime}(s, u)=\left(x(s) L, r(s+l-h) \frac{R}{r_{\max }} u, y(s) L\right)
\end{aligned}
$$

The unknown $h$ is solved numerically using:

$$
\int_{0}^{h} \sqrt{\left(\frac{d x(t)}{d t}\right)^{2}+\left(\frac{d y(t)}{d t}\right)^{2}} d t-\frac{l}{L}=0
$$

\section{Differential form of the dynamic equation}

The dynamic equation allows to fully parametrise a leaf emergence scenario for any stage of development of a leaf. However, it will produce always the same pre-defined mature leaf. The dynamic equation could also be written in a differential form, to allow dynamic transformation of leaf shape during a simulation. To do so, $r(a g e)$ is derived to get $\frac{d r}{\text { dage }}=f($ age $)$.

The variational midrib curve, $\mathcal{N}(a g e, s)$, is computing by interpolating a set of midrib curves with a mature length at different ages. It can be expressed either directly or by its derivative form:

$$
\theta(\text { age }, s)=\frac{\partial \mathcal{N}}{\partial s}(\text { age }, s)
$$

At every time step of the simulation $(d t)$, the user will define independently several functions:

- The rate of progression of the leaf through its potential development curve $\left(\frac{d a g e}{d t}\right)$;

- The length increment $\left(\frac{d l}{d t}\right)$;

- The variation of the radius $\left(\frac{d r}{d a g e}\right)$;

- The variation of the curvature of the midrib $\left(\frac{d \theta}{d a g e}\right)$.

\section{E. Plastic transformations}

The surface can be transiently transformed to mimic responses to environmental stress or the effects of constraints exerted by enrolling leaves. Operators can act on leaf curvature by modifying locally the amount curvature. First $\theta(s)$ is computed and the leaf is divided into intervals. A reduction of angle can be specified for every interval. Such operators can also apply on $s$ and $r(s)$, and provide the user with another way of modeling stresses.

\section{F. Surface computation and reduction}

The surface equation can be evaluated at any number of points to build a detailed 3D mesh. Optional modifier of the surface (undulation and twist) can be de-activated to get a first reduction of the number of triangle.
We also implemented a reduction function that allows for further simplification whilst keeping surface unchanged and minimising simultaneously and optimally the error on $x, y, r$. This allows for keeping a maximum of principal characteristics of the surface (angles and width variation).

The algorithm takes as input the number of polygons desired for representing the surface. We do not use classic surface simplification algorithm (e.g. [43], [44]) but rather simplify the generative curve of the surface while minimising the error on the surface. Simplifying curves rather than surface is much more efficient. This is possible because we know the explicit equation of the surface and thus can compute the quadratic error on the surface when we remove a point on the curve. Moreover, simplifying the generative curves allow to maintain the symmetry of the surface while surface decimation algorithms had a tendency to break it, and thus modify the normal of the surface.

Algorithm is as follow :

- Over discretisation of the generative curve;

- Compute for all $s_{i}$ the error induced if $x_{i}\left(s_{i}\right), y_{i}\left(s_{i}\right)$ and $r_{i}\left(s_{i}\right)$ are discarded;

- Let $P_{i}=\left(x_{i}, y_{i}\right)$ and $R_{i}=\left(s_{i}, r_{i}\right)$ two set of points resulting for the discretisation of he curves. Let $\varepsilon^{x y}$ and $\varepsilon^{r}$ be the error on the $\mathcal{N}(s)$ curve and $r(s)$ curve respectively. The errors on each curves are given by:

$$
\begin{aligned}
\varepsilon_{i}^{x y} & =\operatorname{distance}\left(P_{i}, \overrightarrow{P_{i-1} P_{i+1}}\right) \\
\varepsilon_{i}^{r} & =\operatorname{distance}\left(R_{i}, \overrightarrow{R_{i-1} R_{i+1}}\right)
\end{aligned}
$$

The error on the surface is given by:

$$
\forall i \in] 1, n\left[, \varepsilon_{i}=\sqrt{\left(\varepsilon_{i}^{x y}\right)^{2}+\left(\varepsilon_{i}^{r}\right)^{2}}\right.
$$

- Select a point with the smallest error. Points are first added in a heap queue. When one point is removed, the error of its neighbors is updated and placed in the queue. The algorithm is reiterating until the number of points matches the requested number of points.

- Computation of the surface (triangulation) at the requested point and application of an optional deformation.

\section{G. Implementation as components in OpenAlea}

This package use PlantGL [45] for the 3D representation of the geometry of plants, and NumPy/SciPy packages [46] for interpolation and fitting. The model comes with simple visual interface and demos under VisuAlea (Figure 2).

Main functions are:

- Surface normalization and atlas construction from data

- Leaf element mesh builder with simplification given by one parameter

- Dynamic interface giving shape $=f($ age $, L, R)$

- Dynamic differential interface giving

$$
d S=f(a g e, d s, d r, d \theta)
$$




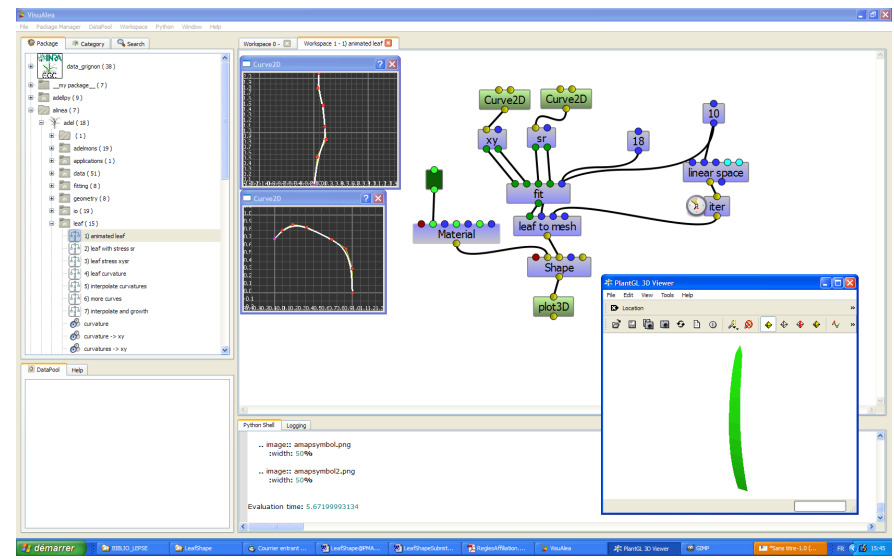

Figure 2. Snapshot of a visualea session running the model. Panels on the left allows for user-interaction in defing midrib curvature and leaf width profile. Leaf is then reconstruct, simplified and transformed into a mesh. a)

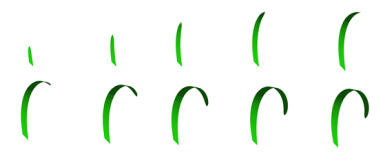

c)

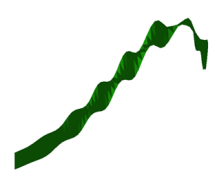

b)

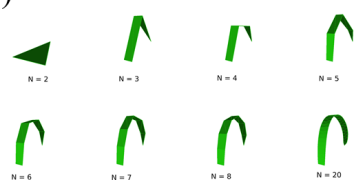

d)

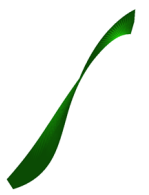

Figure 3. Illustration of some model capabilities. a) Dynamic evolution of the leaf surface; b) Simplification of a leaf with an increasing number of triangles but with the same leaf area; c) Undulation and d) Twist

- Plastic operators on $s, \theta, r$

- Interface interval for applying stress factor along given $s$ intervals

\section{RESUlT}

We will demonstrate the capabilities of our model by showing the plasticity of our leaves and use of the model in three case studies.

\section{A. Plasticity of virtual leaves}

Our model allows to build "multi-plastic" leaves as illustrated on Figure 3. First, our objects have a plasticity in the level of details at which a leaf is represented. Second the evolution of a leaf can be modeled. Finally, the use of morphological operators can add details or transiently affect the shape. The model was also able to capture a variety of shapes as illustrated by sketching of a variety of leaves (Figure 4) coming from a panel of numerised maize and wheat plants [47].
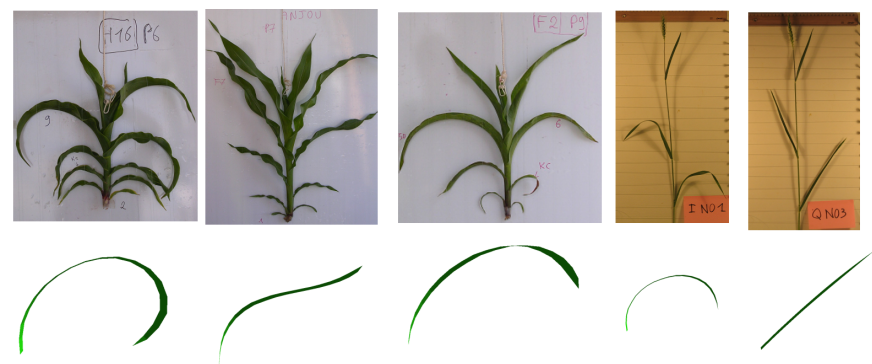

Figure 4. Sketching of different types of gramineaus leaves with the model: 3 varieties of maize contrasting in architecture and 2 varieties of wheat.
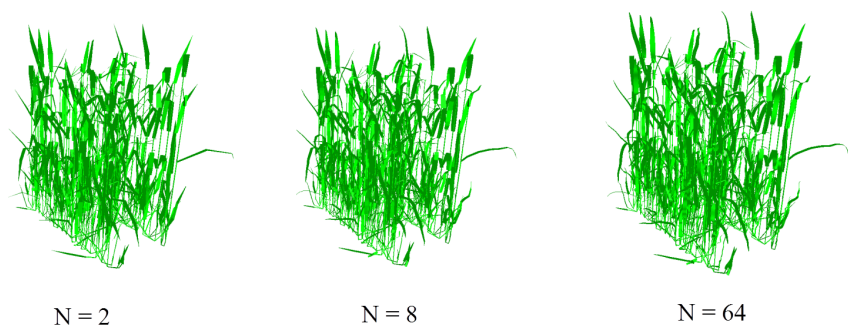

Figure 5. Reconstruction of a small wheat canopy using different levels of discretisation of leaves. Numbers indicate the number of polygon used per leaf.

\section{B. Optimization of computing time}

As stated above, the reducibility of the models allows to optimise computing time by reducing the number of triangle in a scene. We reconstruct with the ADEL-wheat model [27] a small patch of vegetation, using a measured set of leaves with curvature and leaf-width profile. Wheat is at maturity and leaves picked at random in an atlas. We then use the simplification utilities of our model to vary the number of polygons used to represent the leaves (every power of 2 between 2 and 128, Figure 5).

These mock-up were then used as input of a radiative transfert model, that allows computing light interception efficiency [48]. This model allows to replicate the patch, so that the computed value is for a whole canopy. Results show that computation time is a square function of the triangulation level, whilst light interception efficiency rapidly reach an asymptotic value (Figure 6). In this case, an optimal value would be 8 polygons per leaf.

\section{Prediction of leaf shape deformation in response to stresses}

Here we demonstrate how to use the model in a dynamic simulation where leaf shape can be dynamically modified during the simulation. We based on ADEL-maize [24], and test two hypothetic model that mimic the response to two stresses: a) leaf width is responding to local light illumination [5], and b) leaf extension rate and duration are responding to vapour pressure deficit in the air [49]. 


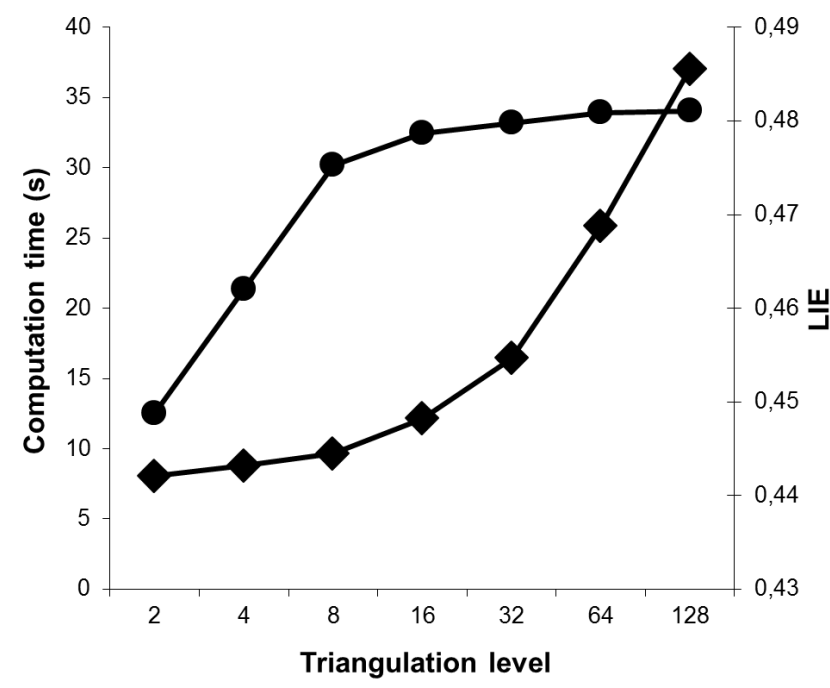

Figure 6. Computation time (diamonds) and light interception efficiency (circle) as a function of the triangulation level of the 3D scene.

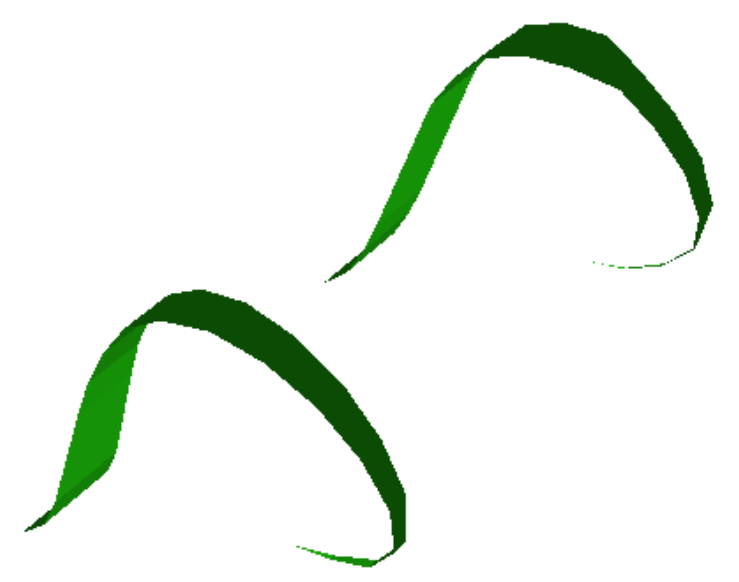

Figure 7. Prediction of leaf shape deformation as a function of response to stresses

Response to light can be modeled as a stress factor affecting the $r($ age) curve. The response to vapour pressure deficit is modeled with a reduction of elongation rate and deceleration of the rate of progression of physiological age with time to mimic an extended duration of elongation. The model allows to predict a deformed shape that can be compared to experiment (Figure 7).

\section{DISCUSSION}

We build an interactive, flexible and ready to use model of gramineous leaf shape. We show that it complies to previously used approaches for modelling leaf geometry in reconstruction or simulation applications. A first important difference is that it could do so all at once, i.e. without having to change the model itself to switch from an approach to another. A second important difference is that the model is not embedded into a particular plant model. We mainly demonstrate here the use with the ADEL family models [24], [27], but also we already use it with success on the rice model ecomeritem [50]. We also add in our package original functionalities, namely the simplification of shapes and a framework to include stresses occurring during growth. The main limitation of our approach is that the important flexibility implies a risk of simulating non realistic shapes. This is particularly true for the modeling of the evolution of curvature during leaf unrolling. Here we demonstrate the ability of the model to handle different scenarios, but all of these have to be parametrised. A further useful step would be to help the user with a less flexible parametrisation based on modeling of mechanical constraints. This step may be a key in our comprehension of gramineous plant development as leaf rolling dynamics within the whorl can mediate important retroaction determining whole plant architecture [51], [52], [31].

\section{ACKNOWLEDGMENT}

This project is supported by Agropolis Fondation in the frame of the OpenAlea project (project 0803-017) and by the Ministère de l'Ecologie et du Développement Durable in the frame of the Echap Project.

\section{REFERENCES}

[1] J. Weber and J. Penn, Creation and rendering of realistic trees. ACM SIGGRAPH, 1995, p. 119128.

[2] B. Lintermann and O. Deussen, "Interactive modeling of plants," IEEE Computer Graphics and Applications, vol. 19, no. 1, pp. 56-65, 1999.

[3] B. Muller, M. Reymond, and F. Tardieu, "The elongation rate at the base of a maize leaf shows an invariant pattern during both the steady-state elongation and the establishment of the elongation zone," Journal of Experimental Botany, vol. 52, no. 359, pp. 1259-1268, 2001.

[4] C. Fournier, "Modélisation des interactions entre plantes au sein des peuplements. application la simulation des régulations de la morphogenèse aérienne du mas (zea mays 1.) par la compétition pour la lumière," Thèse de doctorat, 2000 .

[5] C. Fournier, B. Andrieu, and Y. Sohbi, "Virtual plant models for studying interactions between crops and environment," in Simulation in industry, ESS'2001, N. Giambiasi and C. Frydman, Eds., vol. Erlangen. SCS Europe Bvba, pp. 476-480.

[6] J. Ledent, T. Henkart, and B. Jacobs, "Phénologie du mas, visualisation de la croissance et du développement," Revue de l'Agriculture, vol. 43, no. 3, pp. 391-408, 1990.

[7] B. Moulia, M. Fournier, and D. Guitard, "Mechanics and form of the maize leaf: in vivo qualification of flexural behaviour," Journal of Materials Science, vol. 29, pp. 2359-2366, 1994. 
[8] B. Moulia, "Leaves as shell structures: Double curvature, auto-stresses, and minimal mechanical energy constraints on leaf rolling in grasses," Journal of Plant Growth Regulation, vol. 19, no. 1, pp. 19-30, 2000.

[9] J. O. Hay, B. Moulia, B. Lane, M. Freeling, and W. K. Silk, "Biomechanical analysis of the rolled (rld) leaf phenotype of maize," American Journal of Botany, vol. 87, no. 5, pp. 625-633, 2000.

[10] C. Pradal, S. Dufour-Kowalski, F. Boudon, C. Fournier, and C. Godin, "Openalea: a visual programming and componentbased software platform for plant modelling," Functional Plant Biology, vol. 35, no. 9/10, pp. 751-760, 2008.

[11] R. Bonhomme and C. Varlet-Grancher, "Estimation of the gramineous crop geometry by plant profiles including leaf width variations," Photosynthetica, vol. 12, no. 2, pp. 193196, 1978.

[12] J. Sanderson, T. Daynard, and M. Tollenaar, "A mathematical model of the shape of corn leaves." Canadian Journal of Plant Science, vol. 61, pp. 1009-1011, 1981.

[13] L. Prévot, F. Aries, and P. Monestiez, "Modélisation de la structure géométrique du mas," Agronomie, vol. 11, pp. 491503, 1991.

[14] J. Evers, J. Vos, C. Fournier, B. Andrieu, M. Chelle, and P. Struik, "An architectural model of spring wheat: evaluation of the effects of population density and shading on model parameterization and performance," Ecological Modelling, vol. 200, pp. 308-320, 2007.

[15] T. Dornbusch, J. Watt, R. Baccar, C. Fournier, and B. Andrieu, "A comparative analysis of leaf shape of wheat, barley and maize using an empirical shape model," Annals of Botany, vol. 107, pp. 865-873, 2011.

[16] T. Watanabe, J. S. Hanan, P. Room, T. Hasegawa, H. Nakagawa, and W. Takahashi, "Rice morphogenesis and plant architecture: Measurement, specification and the reconstruction of structural development by $3 \mathrm{~d}$ architectural modelling," Ann Bot, vol. 95, no. 7, pp. 1131-1143, 2005.

[17] D. W. Stewart and L. M. Dwyer, "Mathematical characterization of maize canopies," Agricultural and Forest Meteorology, vol. 66, pp. 247-265, 1993.

[18] F. Aries, "Modélisation surfacique de la structure d'un couvert végétal pour l'étude du rayonnement," Thèse de doctorat, 1997.

[19] M. España, "Simulation de la variation temporelle, directionnelle et spectrale de la réflectance de cultures de mas partir d'un modèle dynamique de la structure $3 \mathrm{~d}$ du couvert," Thèse de doctorat, 1997.

[20] M. España, F. Baret, F. Aries, and B. Andrieu, "Sensitivity of radiative transfer variables calculation to the accuracy of canopy structure description. the case of maize canopy as described by a 3d architecture model," Agronomie, vol. 19, pp. 241-254, 1999.
[21] M. L. España, F. Baret, F. Aries, M. Chelle, and L. Prevot, "Modeling maize canopy 3d architecture - application to reflectance simulation," Ecological Modelling, vol. 122, no. 1-2, pp. 25-43, 1999.

[22] N. Ivanov, P. Boissard, M. Chapron, and B. Andrieu, "Computer stereo plotting for 3-d reconstruction of a maize canopy," Agricultural and Forest Meteorology, vol. 75, pp. 85-102, 1995.

[23] J. L. Drouet, "Modica and modanca: modelling the threedimensional shoot structure of graminaceous crops from two methods of plant description," Field Crops Research, vol. 83, no. 2, pp. 215-222, 2003.

[24] C. Fournier and B. Andrieu, "A 3d architectural and processbased model of maize development," Annals of Botany, vol. 81, no. 2, pp. 233-250, 1998.

[25] P. Kaitaniemi, P. Room, and J. Hanan, "Architecture and morphogenesis of grain sorghum, sorghum bicolor (1.) moench," Field Crops Research, vol. 61, pp. 51-60, 1999.

[26] P. Wernecke, G. Buck-Sorlin, and W. Diepenbrock, "Combining process- with architectural models: The simulation tool vica," Systems Analysis Modelling Simulation, vol. 39, pp. 235-277, 2000.

[27] C. Fournier, B. Andrieu, S. Ljutovac, and S. Saint-Jean, ADEL-wheat: a $3 D$ architectural model of wheat development, ser. Proceedings - PMA03, 2003' International Symposium on plant Growth Modeling, Simulation, visualization and their Applications. Beijing, China, october 13-16, 2003. Beijing, China: Tsinghua University Press and Springer, 2003, pp. 54-66.

[28] J. B. Evers, J. Vos, C. Fournier, B. Andrieu, M. Chelle, and P. C. Struik, "Towards a generic architectural model of tillering in gramineae, as exemplified by spring wheat (triticum aestivum)," New Phytologist, vol. 166, no. 3, pp. 801-812, 2005.

[29] E. Ford, A. Cocke, L. Horton, M. Fellner, and E. Van Volkenburgh, "Estimation, variation and importance of leaf curvature in zea mays hybrids," Agricultural and Forest Meteorology, vol. 148, pp. 1598-1610, 2008.

[30] P. Lewis, The botanical plant modelling system (BPMS). Thiverval-Grignon: INRA Unité EGC, 1997, pp. 45-53.

[31] A. Verdenal, D. Combes, and A. Escobar-Gutiérez, "A study of ryegrass architecture as a self-regulated system, using functional-structural plant modelling," Functional Plant Biology, vol. 35, no. 9/10, pp. 911-924, 2008.

[32] P. Prusinkiewicz, "Art and science for life: Designing and growing virtual plants with 1-systems," Acta Horticulturae, vol. 630, pp. 15-28, 2004.

[33] Y. Guo, Y. Ma, Z. Zhan, B. Li, M. Dingkuhn, D. Luquet, and P. De Reffye, "Parameter optimization and field validation of the functional-structural model greenlab for maize," Annals of Botany, vol. 97, no. 2, pp. 217-230, 2006. 
[34] C. Bassette and F. Bussiere, "3-d modelling of the banana architecture for simulation of rainfall interception parameters," Agricultural and Forest Meteorology, vol. 129, no. 1-2, pp. 95-100, 2005.

[35] A. Peyrat, O. Terraz, S. Merillou, and E. Galin, "Generating vast varieties of realistic leaves with parametric 2gmap 1systems," The visual Computer, vol. 24, no. 7, pp. 807-816, 2008.

[36] J. Ledent, Generation of 3D representations of maize canopies from simple measurements : a tool for visualization or use with models involving plant architecture. Beijing, China: IEEE computer society, 2006, pp. 282-285.

[37] M. España, F. Baret, M. Chelle, F. Aries, and B. Andrieu, "A dynamic model of $3 \mathrm{~d}$ architecture: application to the parameterisation of the clumpiness of the canopy," Agronomie, vol. 18, pp. 609-626, 1998.

[38] N. Goel, L. Knox, and M. Norman, "From artificial life to real life : computer simulation of plant growth," International Journal of General Systems, vol. 18, pp. 291-319, 1990.

[39] W. Wang and B. Joe, "Robust computation of the rotation minimizing frame for sweep surface modeling," ComputerAided Design, vol. 29, no. 5, pp. 379 - 391, 1997. [Online]. Available: http://www.sciencedirect.com/science/ article/pii/S0010448596000772

[40] T. Dornbush, J. Watt, R. Baccar, C. Fournier, and B. Andrieu, Towards a quantitative evaluation of cereal lamina shape using an empirical shape model. Beijing, China: IEEE Computer Society, 2010, pp. 229-236.

[41] H. Sinoquet, B. Moulia, and R. Bonhomme, "Estimating the three-dimensional geometry of a maize crop as an input of radiation models: comparison between three-dimensional digitizing and plant profiles," Agricultural and Forest Meteorology, vol. 55, pp. 233-249, 1991.

[42] D. Barthélémy and Y. Caraglio, "Plant architecture: A dynamic, multilevel and comprehensive approach to plant form, structure and ontogeny," Annals of Botany, vol. 99, no. 3, pp. 375-407, 2007.

[43] M. Garland and P. S. Heckbert, Surface simplification using quadric error metrics. ACM SIGGRAPH, 1997, p. 209216.

[44] M. Garland and Y. Zhou, "Quadric-based simplification in any dimension," ACM Trans. Graph., vol. 24, no. 2, pp. 209-239, 2005.

[45] C. Pradal, F. Boudon, C. Nouguier, J. Chopard, and C. Godin, "Plantgl: a python-based geometric library for 3d plant modelling at different scales," Graphical Models, vol. 71, no. 1, pp. 1-21, 2009.

[46] Scipy, Scientific Tools for Python. Enthought, 2012.

[47] P. Noblet, "Analyse de la variabilité génotypique de l'architecture 3d du mas," Mémoire de stage d'initiation la recherche, 2004.
[48] M. Chelle and B. Andrieu, "The nested radiosity model for the distribution of light within plant canopies," Ecological Modelling, vol. 111, pp. 75-91, 1998.

[49] K. Chenu, S. C. Chapman, G. L. Hammer, G. McLean, H. B. H. Salah, and F. Tardieu, "Short-term responses of leaf growth rate to water deficit scale up to whole-plant and crop levels: an integrated modelling approach in maize," Plant, Cell \& Environment, vol. 31, no. 3, pp. 378-391, 2008.

[50] J.-C. Soulié, C. Pradal, C. Fournier, and D. Luquet, Feedbacks between plant microclimate and morphogenesis in fluctuating environment: analysis for rice using Ecomeristem model coupled with $3 D$ plant and energy balance computation tools in OpenAlea platform. Plant Science Department, University of California, Davis, 2010, pp. 123-125.

[51] C. Fournier, J. L. Durand, S. Ljutovac, R. Schaufele, F. Gastal, and B. Andrieu, "A functional-structural model of elongation of the grass leaf and its relationships with the phyllochron," New Phytologist, vol. 166, no. 3, pp. 881-894, 2005.

[52] B. Andrieu, J. Hillier, and C. Birch, "Onset of sheath extension and duration of lamina extension are major determinants of the response of maize lamina length to plant density," Annals of Botany, vol. 98, no. 5, pp. 1005-1016, 2006. 